\title{
Barium hexaferrite/muscovite heteroepitaxy with mechanically robust perpendicular magnetic anisotropy
}

\author{
Wei-En Ke ${ }^{1}$, Pao-Wen Shao ${ }^{1}$, Chang-Yang Kuo ${ }^{2,3}$, Haili Song ${ }^{4}$, Rong Huang $\mathbb{D}^{5}$, Naoki Yagi ${ }^{6}$, Tsuyoshi Kimura ${ }^{6}$, Yugandhar Bitla $\mathbb{D}^{7}$,
} Chun-Fu Chang (iD $^{8}$ and Ying-Hao Chu (iD ${ }^{1,2 凶}$

Recent advances in the design and development of magnetic storage devices have led to an enormous interest in materials with perpendicular magnetic anisotropy (PMA) property. The past decade has witnessed a huge growth in the development of flexible devices such as displays, circuit boards, batteries, memories, etc. since they have gradually made an impact on people's lives. Thus, the integration of PMA materials with flexible substrates can benefit the development of flexible magnetic devices. In this study, we developed a heteroepitaxy of $\mathrm{BaFe}_{12} \mathrm{O}_{19}(\mathrm{BaM}) /$ muscovite which displays both mechanical flexibility and PMA property. The particular PMA property was characterized by vibrating sample magnetometer, magnetic force microscopy, and $\mathrm{x}$-ray absorption spectroscopy. To quantify the PMA property of the system, the intrinsic magnetic anisotropy energy density of $\sim 2.83 \mathrm{Merg}^{-3}$ was obtained. Furthermore, the heterostructure exhibits robust PMA property against severe mechanical bending. The findings of this study on the BaM/muscovite heteroepitaxy have several important implications for research in next-generation flexible magnetic recording devices and actuators.

npj Flexible Electronics (2021)5:33; https://doi.org/10.1038/s41528-021-00130-y

\section{INTRODUCTION}

The demand for high-density recording devices has been increasing over the past few decades. Among these recording devices, magnetic ones have become the potential candidates due to several technological breakthroughs ${ }^{1}$. In comparison to conventional longitudinal recording, perpendicular recording using a medium with perpendicular magnetic anisotropy (PMA) property has attracted much attention because of its high storage density capability. Normally, the PMA feature of thin film (strong out-of-plane magnetization) originates from the interfacial magnetic anisotropy and magnetocrystalline anisotropy. Magnetic systems with PMA feature has been observed in ferromagnetic multilayers $^{2}$, crystalline alloys ${ }^{3}$, amorphous rare earth-transition metal alloys ${ }^{4}$, and $\mathrm{CoFeB} / \mathrm{MgO}^{5}$ based systems. Among them, barium hexaferrite $\left(\mathrm{BaFe}_{12} \mathrm{O}_{19}, \mathrm{BaM}\right)$ is one of the most promising PMA materials due to its fairly large uniaxial magnetocrystalline anisotropy $\left(>10^{6} \mathrm{erg} \mathrm{cm}^{-3}\right)$, high Curie temperature $(\sim 726 \mathrm{~K})$, and excellent corrosion resistance. In bulk BaM, $\mathrm{Fe}^{3+}$ (High Spin, $\mathrm{d}^{5}$ ) ion centers in oxygen coordination are ferromagnetically coupled. Owing to its robust room temperature hard magnetism, high packing density, resilience to thermal demagnetization, corrosion, and humidity ${ }^{6}$, BaM has found applications in permanent magnets, magnetic card strips, motors, generators, speakers, magnetic tapes, and long term data storage ${ }^{7}$. Insulating magnets like BaM with advantages of the high transition temperature, high coercivity and high magnetic anisotropy field $\left(\mathrm{H}_{\mathrm{K}} \sim 17 \mathrm{kOe}\right)$ with excellent chemical stability and corrosion resistance can pave way towards pure-spin current based spintronic devices ${ }^{8}$. Most striking features of BaM like PMA and low damping are promising for spintronic functionalities like low-power spin-orbit torque switching, high-speed domain-wall motion, high-frequency spinorbit torque oscillation, logical devices, etc ${ }^{9}$. The PMA in BaM thin films originates from the intrinsic anisotropy imposing small constraints on film thickness unlike FM metals like $\mathrm{CoFeB} / \mathrm{MgO}$ which needs to be very thin to exploit interfacial $P M A^{10}$. Therefore, BaM has considerable potential for the development of memory.

Substrates with mechanical flexibility are of great importance due to the increasing demand for functional flexible devices. For instance, metal foils, ultra-thin glass, and polymers are commonly used in the fabrication of these devices. However, opacity of metallic foils hinder their use in optoelectronics, ultra-thin glass is fragile and costly while polymers can't withstand multiple thermal processes. Therefore, a suitable flexible substrate is required. Muscovite mica, a well-known 2D layered oxide, is adopted due to its high mechanical flexibility, optical transparency, and excellent thermal and chemical stabilities. In recent years, numerous studies have validated the growth of high-quality oxide heteroepitaxy on muscovite ${ }^{11-13}$. The heteroepitaxy of the film and substrate is crucial because it is beneficial for us to fully understand the effect of anisotropy, interface, etc. of the whole structure. With the above excellent properties and the ability to fabricate the high-quality oxide heteroepitaxy, muscovite becomes the best template for deposition.

In this study, we fabricated epitaxial yet high-quality BaM/ muscovite heterostructure with PMA property via pulsed laser deposition (PLD). The PMA property was characterized by vibrating sample magnetometer, magnetic force microscopy, and x-ray absorption spectroscopy. The measured saturation magnetization and estimated intrinsic magnetic anisotropy energy density are consistent with the bulk values corroborating the high

\footnotetext{
${ }^{1}$ Department of Materials Science and Engineering, National Yang Ming Chiao Tung University, Hsinchu 30010, Taiwan. ${ }^{2}$ Department of Electrophysics, National Yang Ming Chiao Tung University, Hsinchu 30010, Taiwan. ${ }^{3}$ National Synchrotron Radiation Research Center (NSRRC), 101 Hsin-Ann Road, Hsinchu 30076, Taiwan. ${ }^{4}$ Key Laboratory of Bioinorganic and Synthetic Chemistry of Ministry of Education, School of Chemistry, Sun Yat-Sen University, Guangzhou 510275, China. ${ }^{5}$ Department of Electronic Engineering, East China

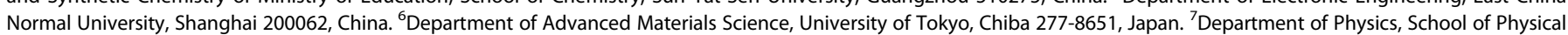
Sciences, Central University of Rajasthan, Ajmer 305817, India. ${ }^{8}$ Max Planck Institute for Chemical Physics of Solids, Nöthnitzer Straße 40, 01187 Dresden, Germany.

凶email: yhc@nctu.edu.tw
} 


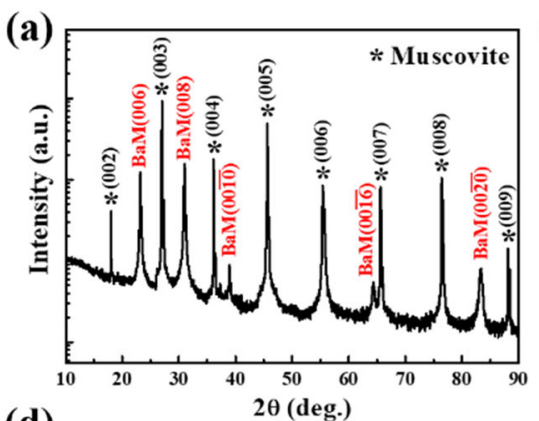

(d)

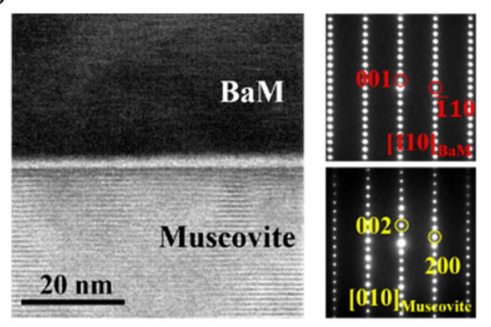

(b)

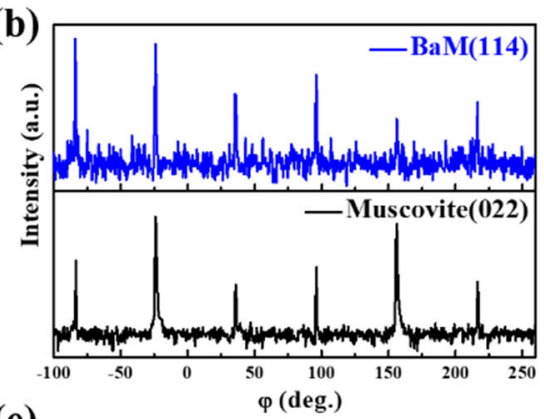

(e)

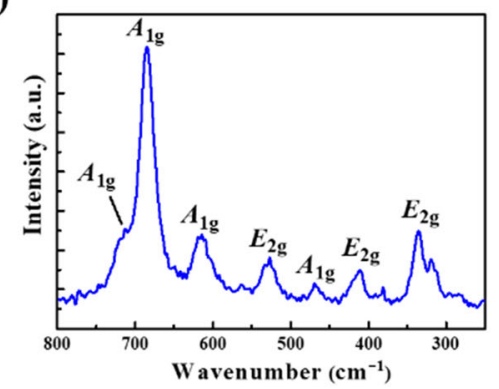

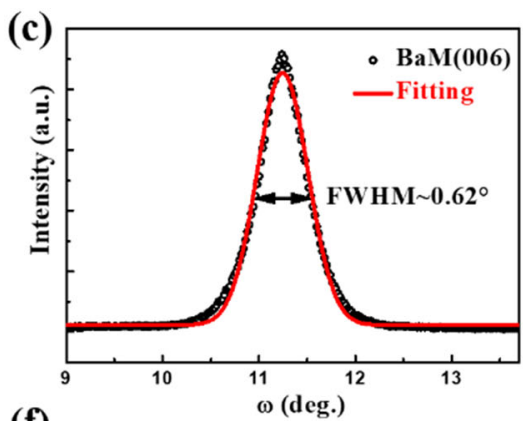

(f)

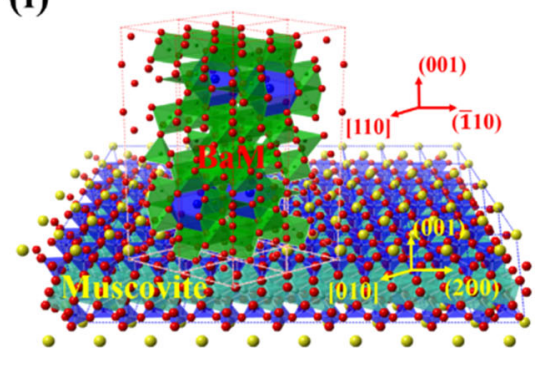

Fig. 1 Structural analyses of the BaM/muscovite heteroepitaxy. a Out-of-plane XRD 2 $\theta-\theta$ scan. b Phi scans of BaM(114) and muscovite(022) reflections. c Rocking curve of $\mathrm{BaM}(006)$ peak. d Cross-sectional TEM image and corresponding selected area electron diffraction patterns. e Raman spectrum with the feature of $A_{1 \mathrm{~g}}$ and $E_{2 \mathrm{~g}}$ modes. $\mathbf{f}$ Schematic of the epitaxial relationship of BaM film and muscovite substrate.

quality of BaM film. Furthermore, the PMA property remains robust even under severe mechanical bending. These results demonstrate that $\mathrm{BaM} /$ muscovite heteroepitaxy can lead to potential applications in flexible magnetic recording devices and actuators with high-temperature stability in the future.

\section{RESULTS AND DISCUSSION}

\section{Structural analyses}

Recently, muscovite mica has been suggested as an excellent platform for the growth of oxide heteroepitaxy ${ }^{13}$. This motivated us to adopt muscovite as the substrate to grow BaM film to obtain PMA. To confirm the heteroepitaxy of BaM/muscovite, the heterostructure was examined by X-ray diffraction (XRD). A typical $2 \theta-\theta$ scan (Fig. 1a) shows that (001)-oriented BaM film is grown on (001)-oriented muscovite substrate along the out-of-plane (OOP) direction without any secondary phase. To further determine the epitaxial relationship between film and substrate, the phi scans were employed. As shown in Fig. $1 \mathrm{~b}$, the perfect alignment between the muscovite(022) and $\operatorname{BaM}(114)$ peaks at every $60^{\circ}$ interval indicates the in-plane (IP) epitaxial relationship as BaM [110]//muscovite[010]. The full width at half maximum (FWHM) of $\mathrm{BaM}(006)$ peak is $\sim 0.62^{\circ}$ as shown in Fig. 1c. The result indicates a better crystallinity of BaM film compared to that on other rigid substrates ${ }^{14}$. To gain more insights into the crystal orientation and reveal the $\mathrm{BaM} /$ muscovite interface, a cross-sectional transmission electron microscopy was performed, and the result is shown in Fig. 1d. The selected area electron diffraction patterns of BaM film (upper-right) and muscovite substrate (lower-right) are very sharp, indicating the good crystallinity of BaM film and substrate. The epitaxial relationships determined by the electron diffraction are $(001)_{\mathrm{BaM}} / /(001)_{\text {Muscovite }}$ and $[110]_{\mathrm{BaM}} / /[010]_{\text {Muscovite, }}$ consistent with the XRD results. However, an ultra-thin amorphous layer is evident at the film-substrate interface that accommodates a large lattice mismatch between BaM film and muscovite substrate. A similar observation was made in high-quality $\mathrm{CoFe}_{2} \mathrm{O}_{4} /$ muscovite heterostructure $^{15}$. Thus, it is reasonable to expect that this layer acts as a structure transition layer for the coherent growth of BaM during the deposition process. To further investigate the crystal symmetry and atomic arrangement of BaM, Raman spectroscopy was employed and the result is shown in Fig. 1e. The peaks of $A_{1 \mathrm{~g}}$ and $E_{2 g}$ are identified. According to the study of Kreisel et al. ${ }^{16}$, BaM crystal can be stacked into $Z(X Y) \bar{Z}, X(Y Z) \bar{X}, X(Z Z) \bar{X}$, and $Z(Y Y) \bar{Z}$ structures. Upon comparison, a structure of $Z(Y Y) \bar{Z}$ BaM is confirmed in our case. In addition, the strongest Raman mode at $684 \mathrm{~cm}^{-1}\left(A_{1 \mathrm{~g}}\right)$ can be allocated to the motion of the $\mathrm{FeO}_{5}$ bipyramidal group. A schematic of heteroepitaxy with the orientation relationship is shown in Fig. 1f. This result meets our expectation because [001] is the easy magnetization axis of $\mathrm{BaM}$, which results in the desired PMA property of the system. All these experimental results provide crucial evidence on the growth of high-quality $\mathrm{BaM} /$ muscovite heterostructure.

\section{Magnetic properties}

After the establishment of the heteroepitaxy, attention is paid to the magnetic properties. A schematic of the BaM magnetic structure is shown in Fig. $2 \mathrm{a}$ wherein $\mathrm{Fe}^{3+}$ ions are situated in five different crystallographic positions with tetrahedral, octahedral, and bipyramidal oxygen coordinations. Because of the magnetocrystalline anisotropy, there exists an easy axis of magnetization along [001] in the structure and the theoretical magnetic moment per formula unit is $20 \mu_{B}{ }^{17}$. Room temperature IP and OOP magnetic hysteresis loops of $\mathrm{BaM} /$ muscovite heterostructure were measured by a vibrating sample magnetometer (VSM). From Fig. $2 b$, it is evident that the easy axis of magnetization lies in the OOP direction, confirming the PMA property of the structure. Additionally, the value of saturation magnetization $\left(19.2 \mu_{B}\right.$ f.u. ${ }^{-1}$ at $30 \mathrm{kOe}$ ) is the best among reported BaM films (Table 1) but slightly smaller than the theoretical value $\left(20 \mu_{B}\right.$ f.u. $\left.{ }^{-1}\right)$. Since $20 \mu_{B}$ f.u. ${ }^{-1}$ was assumed in pure $\mathrm{BaM}$ at $0 \mathrm{~K}$, it is reasonable to get the lower value measured at room temperature. The deduction of IP saturation magnetization is believed to be derived from the strong effect of magnetocrystalline anisotropy caused by BaM crystal. To confirm whether the sample is saturated or not, a larger magnetic field of $50 \mathrm{kOe}$ was applied and the result is shown in Supplementary Information Fig. 1. However, the sample does not saturate to the same value in IP and OOP directions due to the strong intrinsic magnetocrystalline anisotropy of BaM crystal. For 
(a)

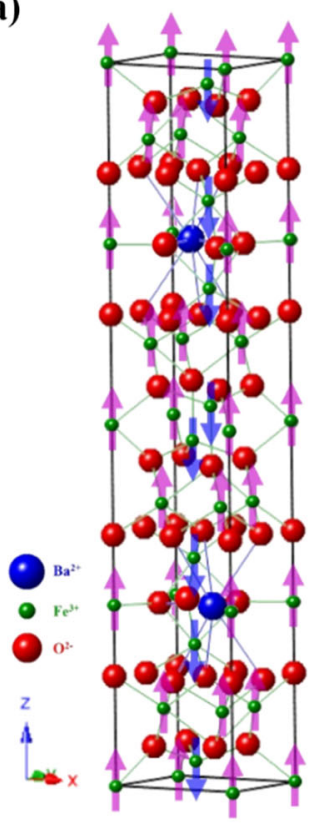

(b)

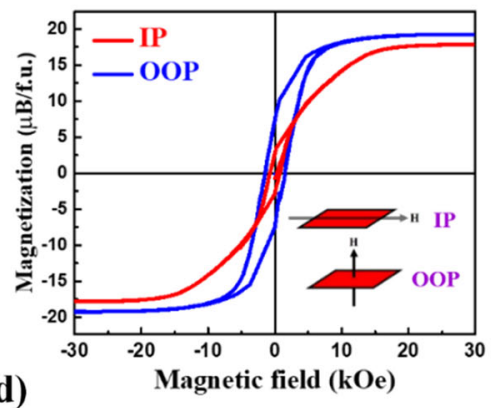

(d)

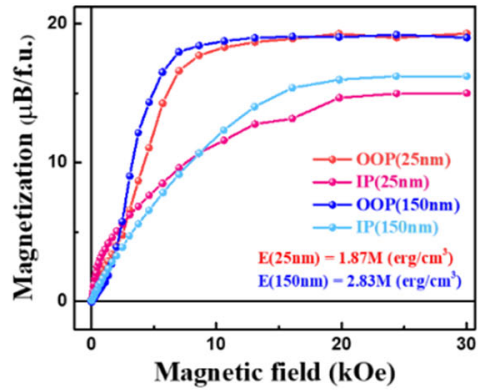

(c)

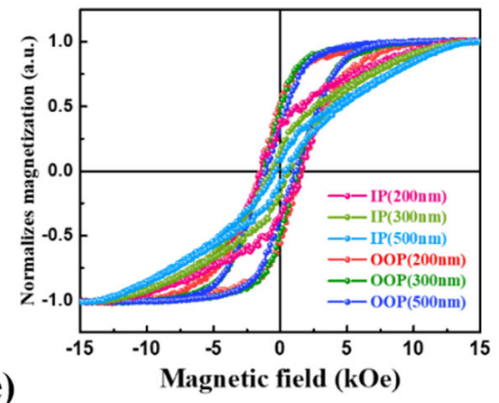

(e)

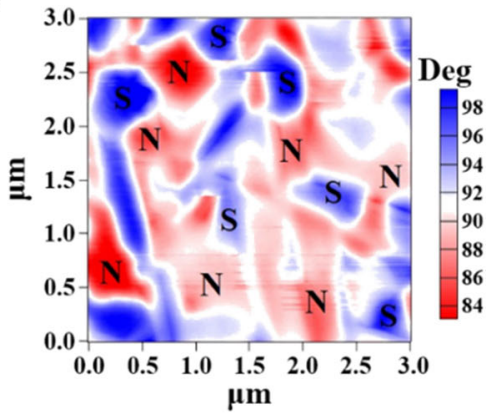

Fig. 2 Magnetic properties. a Schematic of BaM magnetic structure. b Hysteresis loops showing the characteristic of PMA. c IP and OOP hysteresis loops with different thicknesses. d Calculation of intrinsic anisotropy energy density with film thicknesses of $25 \mathrm{~nm}$ and $150 \mathrm{~nm}$. e MFM image.

the thin-film structures, the competition between the magnetocrystalline and shape anisotropy determines the PMA behavior. Thus, the effect of film thickness on PMA reflecting the variation of the shape anisotropy is also investigated. Figure $2 c$ shows the hysteresis loops for BaM films with three different thicknesses. This denotes that the hard axis of magnetization is prone to lie along the IP direction with the increase in film thickness. Nevertheless, no significant differences were found in the OOP hysteresis loops with the increase in the thickness. Therefore, it is necessary to quantify the intrinsic anisotropy energy density of BaM film. For this, we first demagnetize the sample and then apply a magnetic field to the saturation condition. From the IP and OOP virgin magnetic isotherms presented in Supplementary Information Fig. 2 , we can obtain the intrinsic anisotropy energy density based on the formula as:

$E=A_{1}-A_{2}+2 \pi M_{\mathrm{S}}^{2}$

where $E$ represents the intrinsic anisotropy energy density (unit: erg $\left.\mathrm{cm}^{-3}\right), A_{1}$ and $A_{2}$ are the integral areas of IP and OOP $M(H)$ curves, respectively (unit: erg $\mathrm{cm}^{-3}$ ), and the term $2 \pi M_{S}^{2}$ is the energy density of shape anisotropy contributed by the thin film. From the calculation, the intrinsic anisotropy energy density of $\mathrm{BaM}$ /muscovite heteroepitaxy is $\sim 2.83 \times 10^{6} \mathrm{erg} \mathrm{cm}^{-3}$ which is larger than the value of $\mathrm{BaM} / \mathrm{SiO}_{2}{ }^{18}$ and polycrystalline $\mathrm{BaM} / \mathrm{Si}^{19}$. Based on the above calculation, we then provide concrete evidence that the PMA increases with film thickness increasing from $25 \mathrm{~nm}$ to $150 \mathrm{~nm}$, and the result is shown in Fig. $2 \mathrm{~d}$. To understand this fascinating behavior microscopically, magnetic force microscopy (MFM) was utilized to probe the magnetic domains of the sample surface. Typically, MFM is sensitive to the OOP magnetic moment. Based on the results shown in Fig. 2e, the distribution of magnetic domains mainly on OOP direction with opposite contrasts can be noticed, and the average size of the magnetic domain is within $1 \mu \mathrm{m}$. Such a small domain size can be the possible reason for lower saturated magnetic moments. All these results indicate that the PMA properties of BaM/muscovite based on microscopic and macroscopic evidences are attributed to crystalline anisotropy. A comparison between our results and the literature on BaM film is shown in Table 1. It can be seen that our heteroepitaxial system with flexibility shows comparable performance on magnetic anisotropy and saturation magnetization compared to either polycrystalline BaM film or other epitaxial BaM systems on single crystalline substrates.

\section{XAS-XMCD measurements}

Since the magnetism of the structure is attributed to the contribution of Fe ions, it is necessary to determine the valence state of $\mathrm{Fe}$ ions and its contribution along different directions. Therefore, element-specific x-ray absorption spectroscopy (XAS) and $x$-ray magnetic circular dichroism (XMCD) were employed. As shown in Fig. $3 a$, the Fe $L_{2,3}$ XAS spectrum of BaM film taken at room temperature is compared to those of $\mathrm{Fe}_{2} \mathrm{O}_{3}$ and $\mathrm{Fe}_{0.04} \mathrm{Mg}_{0.96} \mathrm{O}^{20}$ serving as the references for $\mathrm{Fe}^{3+}$ and $\mathrm{Fe}^{2+}$ ions, respectively. The lowest energy feature of $\mathrm{Fe}_{0.04} \mathrm{Mg}_{0.96} \mathrm{O}$ at $\mathrm{L}_{3}(705.6 \mathrm{eV})$ is characteristic of $\mathrm{Fe}^{2+}$ which has energy well below that of the $\mathrm{Fe}^{3+}$ feature, indicating BaM film is free of $\mathrm{Fe}^{2+}$ ions. In addition, the $\mathrm{L}_{3}$ main peak at $709.1 \mathrm{eV}$ of BaM film coincides with that of $\mathrm{Fe}_{2} \mathrm{O}_{3}\left(\mathrm{Fe}^{3+}\right)$ standard reference, which further validates that $\mathrm{Fe}$ ions have a valence state of $3+$. To further determine the orientation of magnetic moment of Fe ions in BaM film, XMCD, the differential XAS between right circularly polarized $\left(\mathrm{RCP}, \mu^{+}\right)$and left circularly polarized $\left(\mathrm{LCP}, \mu^{-}\right)$light at remanent magnetization was carried out through the measurement of the absorption of $\mathrm{Fe}$ $L_{2,3}$ edges as shown in Fig. 3b-e. A distinct XMCD signal was detected, inferring that the magnetic source of this heteroepitaxy is attributed to the $\mathrm{Fe}^{3+}$ ions in the structure. The incident light was introduced at four different grazing angles (Normal $0^{\circ}, 20^{\circ}$, $40^{\circ}, 70^{\circ}$ ). By altering the different incident angles, one can pick up both the OOP and IP magnetic signals. As the grazing angle of the $x$-ray increases, the signal of XMCD decreases, demonstrating that the IP magnetic component is weaker than that of OOP. Such a result supports our expectation, providing further confirmation of the PMA behavior of BaM/muscovite heteroepitaxy. 
4

\section{Bending tests}

One of the key advantages of using muscovite as a growth template is its superior mechanical flexibility. To exhibit the potential for flexible application using the BaM/muscovite heteroepitaxy, various bending tests were carried out and the results are shown in Fig. 4. The bending radii were chosen from infinity (flat) to $3.5 \mathrm{~mm}$ under the flex-in (FI: the left inset of Fig. 4c) and flex-out (FO: the right inset of Fig. 4c) modes. Macroscopic hysteresis loops are illustrated in Fig. $4 \mathrm{a}$ and $4 \mathrm{~b}$. Through prudent sort-out, we discover that the changes in saturation magnetization $\left(\Delta M_{S}\right)$ and remanence $\left(\Delta M_{R}\right)$ are all within $10 \%$ under mechanical bending as shown in Fig. $4 c$, d. The calculations of $\Delta M_{S}$ and $\Delta M_{R}$ are expressed as

$$
\begin{aligned}
& \Delta M_{\mathrm{S}}(\%)=\frac{M_{\mathrm{S}, \text { bent }}-M_{\mathrm{S}, \infty}}{M_{\mathrm{S}, \infty}} \times 100 \\
& \Delta M_{\mathrm{R}}(\%)=\frac{M_{\mathrm{R}, \text { bent }}-M_{\mathrm{R}, \infty}}{M_{\mathrm{R}, \infty}} \times 100
\end{aligned}
$$

where $M_{\mathrm{S}(\mathrm{R}) \text {,bent }}$ and $M_{\mathrm{S}(\mathrm{R}), \infty}$ denote the saturation (remanent) magnetization under bent and flat conditions, respectively. To verify the durability of the heterostructure, a retention test was employed. Figure $4 \mathrm{e}$ shows that the anisotropy energy varies within $10 \%$ over $1000 \mathrm{~h}$ under the flex-out mechanical bending with a radius of $3.5 \mathrm{~mm}$. The cycling test was also utilized to confirm the repeatability of the heteroepitaxy as shown in Fig. 4f. The anisotropy energy remains within $10 \%$ during 1000 cycles in the flex-out bending mode with a radius of $3.5 \mathrm{~mm}$. In addition, the samples with the thickness range from $25 \mathrm{~nm}$ to $400 \mathrm{~nm}$ show similar behaviors. A summary is shown in Table 2 to compare our results with other flexible PMA systems in the literature. One can see that the alloy systems dominate the current PMA due to the nature of superior ability of deformation. Our results come up with an additional oxide member with excellent thermal stability for potential PMA candidates. These results establish that BaM/ muscovite heteroepitaxy can retain its initial PMA property under external mechanical bending, essential for futuristic flexible magnetic recording devices and actuators.

In summary, we have fabricated epitaxial (001) BaM film on a muscovite substrate by the PLD technique. One key feature of such a combination is the gain of superior thermal stability. The epitaxial relationship between film and substrate has also been confirmed. Due to the specific crystal alignment with muscovite which shows the easy magnetization along [001], the structure exhibits a clear feature of PMA based on the characterization of hysteresis loops. By virtue of tunable element-specific XAS and XMCD, not only the valence state of iron was confirmed but also the precise and concrete evidence of the perpendicular magnetic moment arrays in the film was established. Furthermore, the bending tests affirm high mechanical flexibility and durability of the heterostructure. Among current available substrates, muscovite delivers an outstanding flexible platform for epitaxial growth, which cannot be achieved on other flexible substrates such as glass, metal foil, polymer, etc. Additionally, our combination can deliver a flexible magnetic system with high-temperature stability. Therefore, mechanical strain robustness of BaM/muscovite heteroepitaxy validates its prospects in the design of flexible magnetic recording and actuation applications with enhanced performance metrics.

\section{METHODS}

\section{Sample preparation}

BaM films were deposited on a muscovite(001) substrate by the PLD technique. KrF laser with a wavelength of $248 \mathrm{~nm}$ was used. The energy density of the pulsed laser was $\sim 1 \mathrm{~J} \mathrm{~cm}^{-2}$ with a repetition rate of $30 \mathrm{~Hz}$. During the growth, the deposition temperature was kept at $900^{\circ} \mathrm{C}$ in oxygen pressure of 1.5 Torr. After the deposition, the sample was annealed at $900^{\circ} \mathrm{C}$ in oxygen pressure of 100 Torr for an hour to release the internal stress inside 
(a)

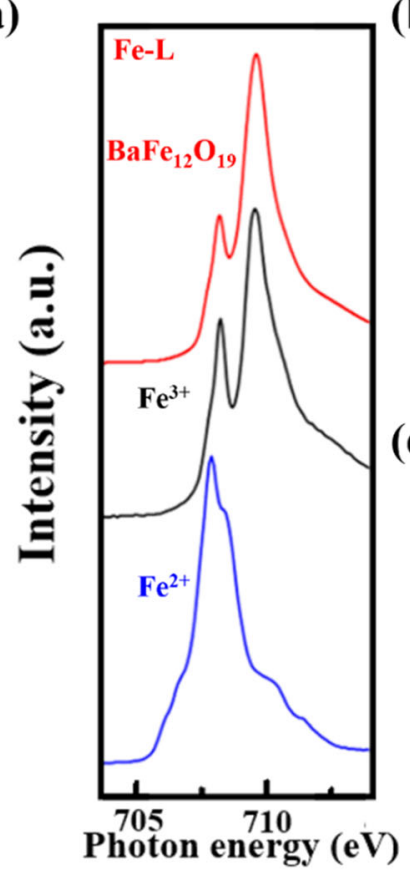

(b)

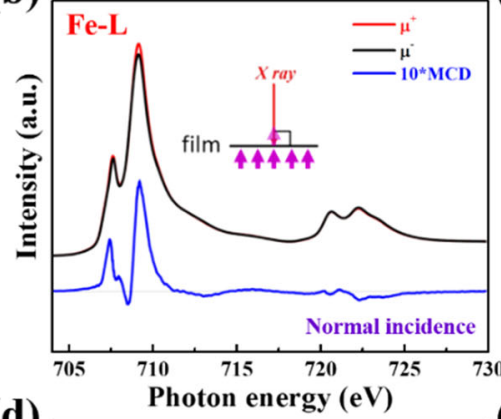

(d)

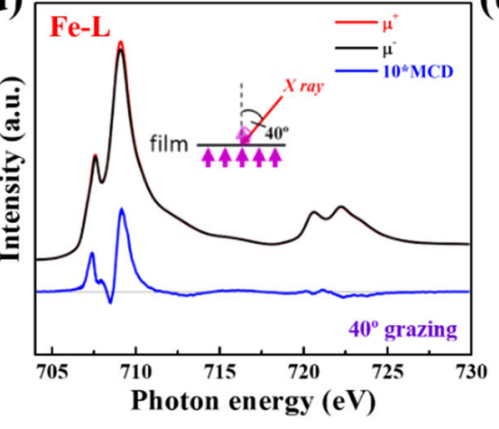

(c)

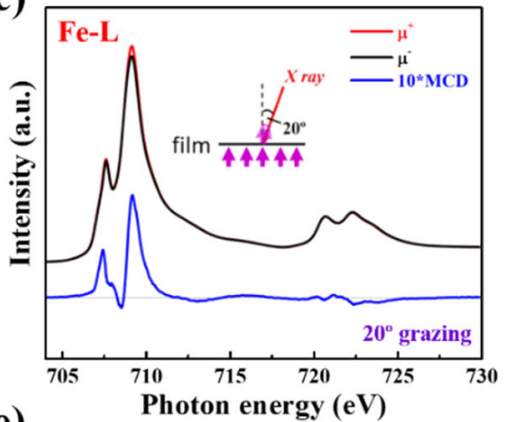

(e)

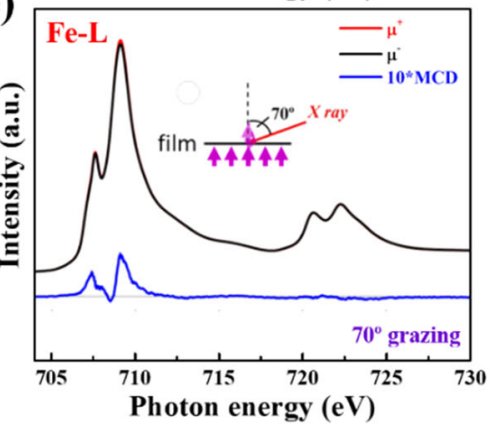

Fig. 3 XAS-XMCD measurements. a XAS of BaM film, reference $\mathrm{Fe}_{2} \mathrm{O}_{3}\left(\mathrm{Fe}^{3+}\right)$, and reference $\mathrm{Fe}_{0.04} \mathrm{Mg}_{0.96} \mathrm{O}\left(\mathrm{Fe}^{2+}\right)$ at $\mathrm{Fe} \mathrm{L}_{3}$ edge taken at room temperature. XAS and corresponding XMCD at Fe $\mathrm{L}_{2,3}$ edge of BaM film measured by incident $\mathbf{x}$-ray at four different grazing angles $\mathbf{b}$ Normal $0^{\circ}$, c $20^{\circ}$, d $40^{\circ}$ and e $70^{\circ}$.

(a)

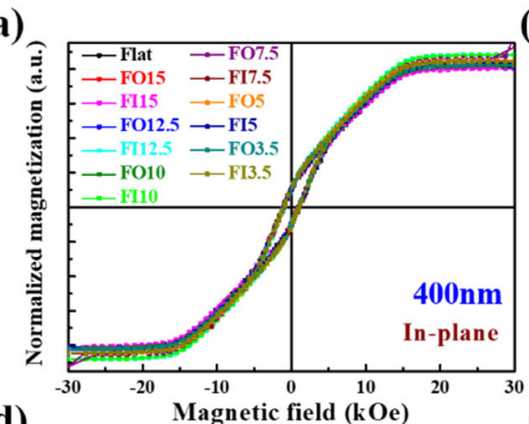

(d)

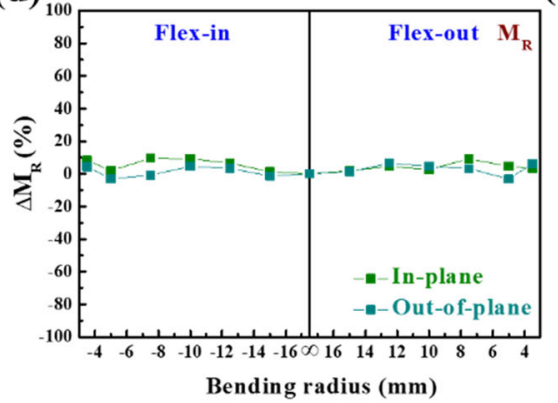

(b)

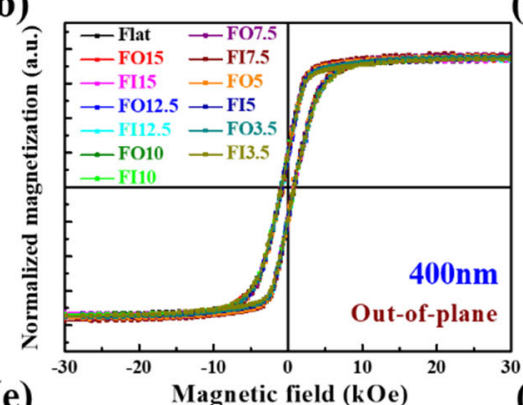

(e) Magnetic field (kOe) (f)

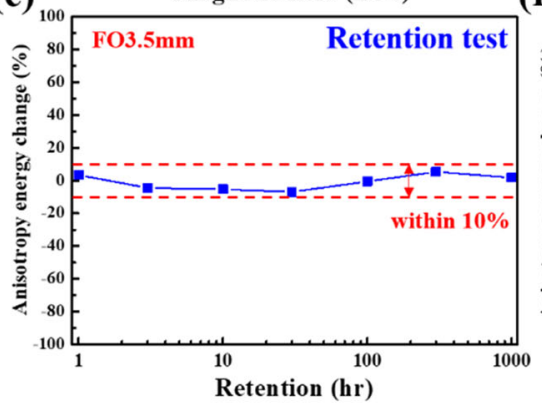

(c)

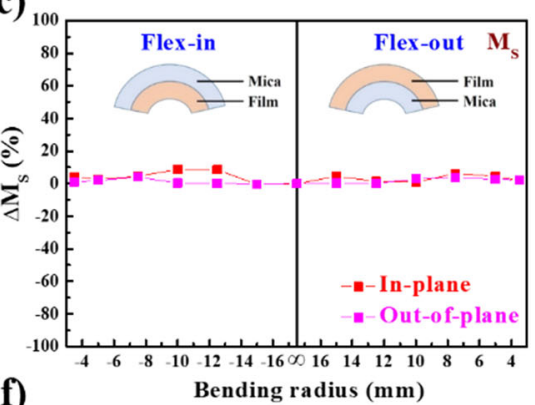

Bending radius $(\mathrm{mm})$

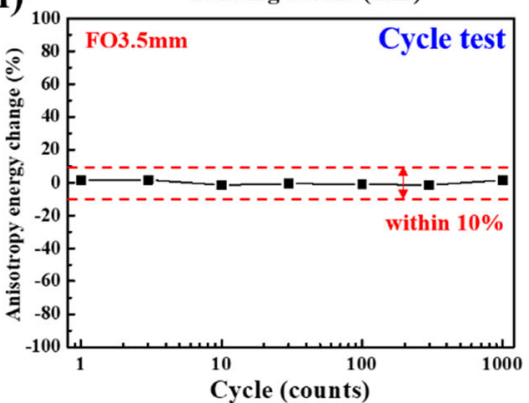

Fig. 4 Bending tests. Hysteresis loops under different bending radii with a IP magnetization and b OOP magnetization. Changes in c saturation magnetization and $\mathbf{d}$ remanence under different bending radii. The insets in $\mathbf{c}$ show two different modes: flex-in (FI) (left), flex-out (FO) (right). e Time and $\mathbf{f}$ bending cycle dependence of the anisotropy energy change for an FO bending radius of $3.5 \mathrm{~mm}$.

BaM film. Afterwards, the film was cooled at the rate of $0.1^{\circ} \mathrm{C} \mathrm{s}^{-1}$ to prevent it from cracking. The thickness of BaM film was varied in this study.

\section{Structural analyses}

X-ray diffraction measurements were performed by Bruker D8 Discover XRD system using monochromatic $\mathrm{Cu} \mathrm{Ka}{ }_{1}$ radiation $(\lambda=1.54056 \AA)$. The crosssectional TEM image was measured by JEOL 2100F. The accelerating voltage was controlled at $200 \mathrm{kV}$. A high-resolution micro-Raman spectrometer (DXR, Thermo
Scientific) equipped with a motorized sample stage was used to acquire the Raman spectrum. To avoid laser-induced heating and damage, the excitation wavelength of $532 \mathrm{~nm}(2.33 \mathrm{eV})$ with power below $0.1 \mathrm{~mW}$ was adopted.

\section{Magnetic properties}

All hysteresis loops were measured by a vibrating sample magnetometer. MFM image was measured by MFP-3D (Asylum Research). PPP-MFMR probe was used. 
Table 2. Comparison of flexible PMA structures.

\begin{tabular}{|c|c|c|c|c|c|}
\hline Structure & Origin of PMA & $\begin{array}{l}\text { Minimum bending } \\
\text { radius }(\mathrm{mm})\end{array}$ & $\begin{array}{l}\text { Endurance } \\
\text { temperature }(\mathrm{K})\end{array}$ & $\begin{array}{l}\text { Anisotropy energy }\left(10^{6}\right. \\
\left.\mathrm{erg} / \mathrm{cm}^{3}\right)\end{array}$ & Ref. \\
\hline $\begin{array}{l}\mathrm{Pt} / \text { ionic gel/Pt/(Co/Pt })_{x} / \mathrm{Ta} / \\
\text { muscovite }\end{array}$ & $\mathrm{Co} / \mathrm{Pt}$ & 5 & $<400^{\mathrm{a}}$ & 3.16 (Bent) & 25 \\
\hline $\mathrm{Ta} / \mathrm{Pt} / \mathrm{CoFeB} / \mathrm{Pt} / \mathrm{PI}$ & $\mathrm{CoFeB} / \mathrm{Pt}$ & $<10$ & $<700$ & Not mentioned ${ }^{\mathrm{b}}$ & 26 \\
\hline $\mathrm{Ta} / \mathrm{MgO} / \mathrm{Co} / \mathrm{Pt} / \mathrm{Ta} / \mathrm{PENC} / \mathrm{PEN}$ & $\mathrm{Co} / \mathrm{Pt}$ & $\pm 20-30$ & $<550$ & Not mentioned ${ }^{c}$ & 27 \\
\hline $\mathrm{Pt} / \mathrm{Co} / \mathrm{Pt} /$ paper & $\mathrm{Co} / \mathrm{Pt}$ & Not mentioned ${ }^{d}$ & $<600$ & Not mentioned ${ }^{\mathrm{e}}$ & 28 \\
\hline BaM/muscovite & Epitaxial BaM film & 3.5 & $<726$ & 2.83 & This work \\
\hline
\end{tabular}

\section{XAS-XMCD measurements}

XAS and XMCD experiments were performed at the NSRRC-MPI TPS 45A Submicron Soft X-ray Spectroscopy beamline at the Taiwan Photon Source in Taiwan. The Fe $L_{2,3}$ spectra were taken in the total electron yield (TEY) method with a photon energy resolution of $50 \mathrm{meV}$ at room temperature. $\mathrm{A} \mathrm{Fe}_{2} \mathrm{O}_{3}$ single crystal was measured simultaneously in a separate chamber to serve as an energy reference for $\mathrm{Fe}_{2,3}$ edge. The XMCD measurements were carried out at remanent magnetization. BaM film was magnetized to magnetic saturation state with $30 \mathrm{kOe}$ along the surface normal of the film before being introduced into the vacuum chamber.

\section{DATA AVAILABILITY}

The data that support the findings of this study are available from the corresponding author upon reasonable request.

Received: 13 July 2021; Accepted: 16 November 2021; Published online: 09 December 2021

\section{REFERENCES}

1. Lin, P. H. et al. Manipulating exchange bias by spin-orbit torque. Nat. Mater. 18, 335-341 (2019).

2. Lairson, B. M., Perez, J. \& Baldwin, C. Application of Pd/Co multilayers for perpendicular magnetic recording. Appl. Phys. Lett. 64, 2891-2893 (1994).

3. Mizukami, S. et al. Fast magnetization precession observed in $L 1_{0}-$ FePt epitaxial thin film. Appl. Phys. Lett. 98, 052501 (2011).

4. Ding, M. \& Poon, J. Tunable perpendicular magnetic anisotropy in GdFeCo amorphous films. J. Magn. Magn. Mater. 339, 51-55 (2013).

5. Ikeda, S. et al. A perpendicular-anisotropy CoFeB-MgO magnetic tunnel junction. Nat. Mater. 9, 721-724 (2010).

6. Martirosyan, K. S., Galstyan, E., Hossain, S. M., Wang, Y. J. \& Litvinov, D. Barium hexaferrite nanoparticles: synthesis and magnetic properties. Mater. Sci. Eng. B-Adv. Funct. Solid-State Mater. 176, 8-13 (2011).

7. Pillai, V., Kumar, P., Multani, M. S. \& Shah, D. O. Structure and magnetic properties of nanoparticles of barium ferrite synthesized using microemulsion processing. Colloid Surf. A-Physicochem. Eng. Asp. 80, 69-75 (1993).

8. Li, P. et al. Generation of pure spin currents via spin Seebeck effect in self-biased hexagonal ferrite thin films. Appl. Phys. Lett. 105, 242412 (2014).

9. Li, P. et al. Spin-orbit torque-assisted switching in magnetic insulator thin films with perpendicular magnetic anisotropy. Nat. Commun. 7, 12688 (2016).

10. Wang, W. X. et al. The perpendicular anisotropy of $\mathrm{CO}_{40} \mathrm{Fe}_{40} \mathrm{~B}_{20}$ sandwiched between $\mathrm{Ta}$ and $\mathrm{MgO}$ layers and its application in $\mathrm{CoFeB} / \mathrm{MgO} / \mathrm{CoFeB}$ tunnel junction. Appl. Phys. Lett. 99, 012502 (2011).

11. Bitla, Y. \& Chu, Y. H. MICAtronics: a new platform for flexible X-tronics. FlatChem $\mathbf{3}$, 26-42 (2017).
12. Jiang, J. et al. Flexible ferroelectric element based on van der Waals heteroepitaxy. Sci. Adv. 3, e1700121 (2017).

13. Wu, P. C. et al. Heteroepitaxy of $\mathrm{Fe}_{3} \mathrm{O}_{4}$ /muscovite: a new perspective for flexible spintronics. ACS Appl. Mater. Interfaces 8, 33794-33801 (2016).

14. Xu, H., Zhang, W., Peng, B. \& Zhang, W. Properties of barium hexaferrite thin films dependent on sputtering pressure. Appl. Surf. Sci. 257, 2689-2693 (2011).

15. Liu, H. J. et al. Flexible heteroepitaxy of $\mathrm{CoFe}_{2} \mathrm{O}_{4} /$ muscovite bimorph with large magnetostriction. ACS Appl. Mater. Interfaces 9, 7297-7304 (2017).

16. Kreisel, J., Lucazeau, G. \& Vincent, H. Raman spectra and vibrational analysis of $\mathrm{BaFe}_{12} \mathrm{O}_{19}$ hexagonal ferrite. J. Solid State Chem. 137, 127-137 (1998).

17. Wartewig, P., Krause, M. K., Esquinazi, P., Rösler, S. \& Sonntag, R. Magnetic properties of $\mathrm{Zn}$ - and Ti-substituted barium hexaferrite. J. Magn. Magn. Mater. 192, 83-99 (1999).

18. Sui, X., Kryder, M. H., Wong, B. Y. \& Laughlin, D. E. Microstructural origin of the perpendicular anisotropy in M-type barium hexaferrite thin films deposited by if magnetron sputtering. IEEE Trans. Magn. 29, 6 (1993).

19. Abuzir, A. R. \& Salman, S. A. Fabrication and micromagnetic modeling of barium hexaferrite thin films by RF magnetron sputtering. Results Phys. 8, 587-591 (2018).

20. Haupricht, T. et al. Local electronic structure of $\mathrm{Fe}^{2+}$ impurities in $\mathrm{MgO}$ thin films: temperature-dependent soft x-ray absorption spectroscopy study. Phys. Rev. B 82, 035120 (2010).

21. Sun, K. et al. Magnetic property and stress study of barium hexaferrite thin films with different structures. J. Alloy. Compd. 663, 645-650 (2016).

22. Geiler, A. L. et al. Atomic scale design and control of cation distribution in hexagonal ferrites. Phys. Rev. Lett. 101, 067201 (2008).

23. Oliver, S. A., Yoon, S. D., Kozulin, I., Chen, M. L. \& Vittoria, C. Growth and characterization of thick oriented barium hexaferrite films on $\mathrm{MgO}$ (111) substrates. Appl. Phys. Lett. 76, 3612 (2000).

24. Chen, Z. et al. Epitaxial growth of M-type Ba-hexaferrite films on MgO (111) \| SiC (0001) with low ferromagnetic resonance linewidths. Appl. Phys. Lett. 91, 182505 (2007).

25. Zhao, S. et al. Low-voltage control of $(\mathrm{Co} / \mathrm{Pt})_{X}$ perpendicular magnetic anisotropy heterostructure for flexible spintronics. ACS Nano 12, 7167-7173 (2018).

26. Vemulkar, T., Mansell, R., Fernández-Pacheco, A. \& Cowburn, R. P. Toward flexible spintronics: perpendicularly magnetized synthetic antiferromagnetic thin films and nanowires on polyimide substrates. Adv. Funct. Mater. 26, 4704-4711 (2016).

27. Lee, O., You, L., Jang, J., Subramanian, V. \& Salahuddin, S. Flexible spin-orbit torque devices. Appl. Phys. Lett. 107, 252401 (2015).

28. Che, W. et al. Critical anomalous hall behavior in $\mathrm{Pt} / \mathrm{Co} / \mathrm{Pt}$ trilayers grown on paper with perpendicular magnetic anisotropy. Appl. Phys. Lett. 104, 262404 (2014).

29. Makushko, P. et al. Flexible magnetoreceptor with tunable intrinsic logic for onskin touchless human-machine interfaces. Adv. Funct. Mater. 31, 2101089 (2021).

\section{ACKNOWLEDGEMENTS}

This work was supported by the Ministry of Science and Technology, Taiwan (grant No. MOST 110-2634-F-009-026) and the Center for Emergent Functional Matter 
Science of National Yang Ming Chiao Tung University from The Featured Areas Research Center Program within the framework of the Higher Education Sprout Project by the Ministry of Education (MOE) in Taiwan. NY and TK were supported by JSPS KAKENHI (grant Nos. JP19H05823 and JP21H04436). Rong Huang was supported by the National Natural Science Foundation of China (Grant No. 61974042). The XAS and remanent XMCD measurements in Taiwan were supported by the Max PlanckPOSTECH-Hsinchu Center for Complex Phase Materials.

\section{AUTHOR CONTRIBUTIONS}

Y.H.C. conceived the original idea of the project. W.E.K. prepared the sample, performed XRD, VSM measurements, bending tests, and wrote the manuscript through contributions of all authors. H.S. and R.H. carried out TEM measurements. P. W.S. carried out MFM measurements. C.Y.K. and C.F.C. carried out XAS-XMCD measurements. N.Y., T.K., and Y.B. contributed to the connection of different analyses. All authors have approved the final version of the manuscript.

\section{COMPETING INTERESTS}

The authors declare no competing interests.

\section{ADDITIONAL INFORMATION}

Supplementary information The online version contains supplementary materia available at https://doi.org/10.1038/s41528-021-00130-y.
Correspondence and requests for materials should be addressed to Ying-Hao Chu.

Reprints and permission information is available at http://www.nature.com/ reprints

Publisher's note Springer Nature remains neutral with regard to jurisdictional claims in published maps and institutional affiliations. Attribution 4.0 International License, which permits use, sharing, adaptation, distribution and reproduction in any medium or format, as long as you give appropriate credit to the original author(s) and the source, provide a link to the Creative Commons license, and indicate if changes were made. The images or other third party material in this article are included in the article's Creative Commons license, unless indicated otherwise in a credit line to the material. If material is not included in the article's Creative Commons license and your intended use is not permitted by statutory regulation or exceeds the permitted use, you will need to obtain permission directly from the copyright holder. To view a copy of this license, visit http://creativecommons. org/licenses/by/4.0/.

C The Author(s) 2021 